\title{
The Influence of IT Systems on the Use of the Earth's Resources
}

\author{
Jurij Paraszczak \\ IBM T.J. Watson Research Centre \\ Yorktown Heights, NY 10598 \\ jurij@us.ibm.com
}

Information technology is so pervasive that estimates of its power usage show that data centers, locations where computing systems are densely packed consume some $2 \%$ of available power in mature economies. Other estimates show that an additional $8 \%$ or so of power is consumed by IT systems which reside in offices and homes, while assessments of power used consumer devices such as mobile phones and PDA's combined with other household appliances while charging or not deployed can add another $6-8 \%$ of total power consumption. As computing power densities have increased from a few tens of watts per square foot to hundreds of watts per square foot considerable attention has been applied to the management of power in IT systems.

Efforts to deal with these power densities includes compute and storage system redesigns to allow power to be controlled and managed, techniques to monitor and understand the distribution of thermal energy in data centers and the beginning of the integration of the grid to the IT based power demands of the data center.

Inasmuch as managing IT based power is an important issue, the same IT can be used to help manage the planet's resources such as electrical power in homes and enterprises, water delivery and distribution to agriculture and industry, flooding and sewage control, road traffic management and road charging, logistics and manufacturing processes and many other activities which use resources and generate waste.

In many situations where resources are used in cities, their usage is barely measured. For example the delivery of electrical power to homes is rarely measured more than once a month. Once the bill is submitted, the consumer has no real idea where the energy is spent and has no ability to plan how to manage that power. An even more critical issue within electrical power distribution networks is the identification of faults, where very little monitoring of the distribution networks results in irate customers being the only source of information as to the location and timing of faults. Similarly water usage by industrial, agricultural and enterprise customers and consumers is not usually monitored at any detailed level and issues such as leakage and contribute to waste and inefficiency.

Once these resource delivery systems become instrumented along with the corresponding consuming systems, the resulting data can be used to manage resources much more efficiently. This talk will address the nascent domain of resource management systems which combine the fields of data acquisition, distribution, modeling optimization and decision support and begin to allow automated systems to manage 
resources within a city. Examples of the use of these integrated systems for traffic management in cities, energy management in data centers, water management in urban and suburban areas, carbon management in the distribution of goods and other situations will be shown. These will be shown to demonstrate the importance of models and their integration into the operation of these instrumented systems. The scope of resource reduction, while maintaining the same levels of service, will show the range of saving which are possible and descriptions of the IT architectures which enable this combination of data flow, models and decisions to be made will be provided. 